\title{
CORONARY ARTERY DISEASE
}

\section{$\lg A$ anticardiolipin antibody is associated with the extent of daily-life ischaemia in patients with chronic coronary artery disease}

\author{
Ignatios Ikonomidis, John Lekakis, Georgia Vamvakou, Sozos Loizou, loanna Revela, Felicita \\ Andreotti, Dimitrios T Kremastinos, Petros Nihoyannopoulos
}

See end of article for authors' affiliations

Correspondence to: Ignatios Ikonomidis, 2nd Cardiology Department, Attikon Hospital University of Athens, Perikleous $19, \mathrm{~N}$ Chalkidona, Athens, 14343 Greece; ignoik@otenet.gr

Accepted 1 November 2006
Background: Circulating anticardiolipin antibodies $(\mathrm{aCL})$ may cause endothelial dysfunction. We investigated whether $\mathrm{aCL}$ are related to platelet activation, thrombin generation and daily-life ischaemia in patients with chronic coronary artery disease (CAD).

Methods: We measured (medians 25th-75th percentile) $\lg G$, $\lg M$, $\lg A$ aCL serum levels (Arbitrary Elisa Units, $A E U)$, prothrombin fragments $(F 1+2, n m o l / l), 24$ h urine excretion of 11 -dehydrothromboxane $B 2$ (1 1DHTXB2, ng/mg creatinine) creatine kinase (CK) and its cardiac isoenzyme CK-MB (IU/I) in 60 patients with angiographically documented CAD and in 40 age and sex matched controls. Patients underwent a $48 \mathrm{~h}$ Holter monitoring for assessment of the number and duration of ischaemic episodes.

Results: Patients had higher $\lg A-a C L$ levels than controls (3.2 vs 2.4 AEU, $p=0.002$ ). Increased IgA-ACA levels were related to increased number and duration of ischaemic episodes $(p<0.01)$. By ANOVA, patients with $\geqslant 10$ ischaemic episodes (3rd tertile) or duration of ischaemia $\geqslant 32 \mathrm{~min}$ (3rd tertile) had higher IgA-aCL than those with lower ischaemic burden (4.95 vs 3 vs $2.5 \mathrm{AEU}, p=0.002$ and 4.9 vs 3 vs $2.5 \mathrm{AEU}, p=0.001$ respectively). Patients with $\geqslant 2$ ischaemic episodes (2nd and 3rd tertile) had higher 11-DHTXB2, than those with minimal ischaemia ( $2<$ episodes, 1 st tertile) $(p=0.001)$. CK and CK-MB were within normal range after Holter monitoring. Receiver operating curve analysis showed a greater area under the curve for $\lg \mathrm{A}-\mathrm{aCL}$ than for 11 -DHTXB2 in predicting severe ischaemia ( $\geqslant 10$ ischemic episodes or $\geqslant 32$ min duration of ischaemia). Conclusion: Increasing $\lg \mathrm{A}-\mathrm{aCL}$ levels are associated with increasing ischemic burden in patients with CAD.
W hile cardiac enzymes are sensitive markers of myocardial necrosis, there are no reliable biomarkers for myocardial ischaemia. Increased production of anticardiolipin antibodies (aCL) has been linked to lipid peroxidation and may cause endothelial dysfunction favouring vasoconstriction. ${ }^{1}$ In this study we examined the relationship between aCL, platelet activation and ischaemia during daily-life activities as assessed by $48 \mathrm{~h}$ Holter monitoring in chronic coronary artery disease (CAD).

\section{METHODS}

We studied 60 patients ( 53 men, 7 women, age $60 \pm 5$ years, UK inhabitants) scheduled for elective coronary artery bypass graft $(\mathrm{CABG})$, with effort angina of more than 1 year's duration, documented exercise-induced ischaemia, and over 70\% stenosis of more than one coronary artery. Exclusion criteria were evidence of inflammatory diseases, renal/liver failure, heart failure and/or ejection fraction less than $50 \%$ or malignant diseases, ECG repolarisation abnormalities, acute coronary events or revascularisation ( $<6$ months) and diabetes. Forty clinically healthy subjects $(34$ men, 6 women, age $59 \pm 13$ years) with normal ECG, echocardiogram and treadmill test served as controls.

The study was approved by the hospital's ethics committee and subjects gave their informed consent.

Patients discontinued antiplatelets for 10 days following the current guidelines for CABG. At the end of this period, patients underwent $48 \mathrm{~h}$ Holter monitoring (HM) (Marquette 8000 system) to assess the number and duration of ischaemic episodes, blood sampling at $24 \mathrm{~h}$ intervals and $24 \mathrm{~h}$ urine collections. Anti-anginal therapy was gradually reduced over $48 \mathrm{~h}$ to avoid rebound ischaemia and then withheld during the
$48 \mathrm{~h}$ HM with the exception of sublingual nitrates. All patients were able to complete the study. ST depression $>0.1 \mathrm{mV}$, $60 \mathrm{msec}$ after the $\mathrm{J}$ point and lasting $>1$ minute, was considered to indicate ischaemia.

IgG, IgM and IgA-aCL (arbitrary ELISA units, AEU) were identified by ELISA as previously described. ${ }^{2}$ Urinary 11-DTXB2 (a marker of platelet activation) was measured by ELISA (Cascade Biochem, Neogen, Reading, UK; sensitivity 0.01 ng/ $\mathrm{ml}$ ) and values were corrected for $24 \mathrm{~h}$ urinary excretion of creatinine (ng/mg creatinine). Creatine kinase (CK) and its myocardial isoenzyme (CK-MB IU/l) were measured as indices of myocardial damage.

\section{Statistical analysis}

In a pilot study of 12 cases and 8 controls we calculated the means and standard deviations of aCL, PF1+2 and 11-DHTXB2 in each subgroup. We assumed that at least a 30\% increase in the levels of the biochemical indices in CAD patients versus normal controls is clinically significant and thus, using a sample size of 40 subjects for the control group, a type (I) error (a) $=0.05$ (two-tailed) and a power of $80 \%$ for the comparisons between patients and $\mathrm{n}$ controls, the sample size for the patients with CAD was calculated to be 60 . Assuming a twofold increase in the biomarkers of patients within the upper tertile of the number of ischaemic episodes during HM compared to those within the lowest tertile, the sample was calculated to be 20 per group. Biomarkers are expressed as medians. Differences were analysed by Wilcoxon signed rank or Mann-Whitney U

Abbreviations: $\mathrm{aCL}$, anticardiolipin antibodies; $\mathrm{AUC}$, areas under the curves; CK, creatine kinase; HM, Holter monitoring; ROC, receiver operating characteristic curve 


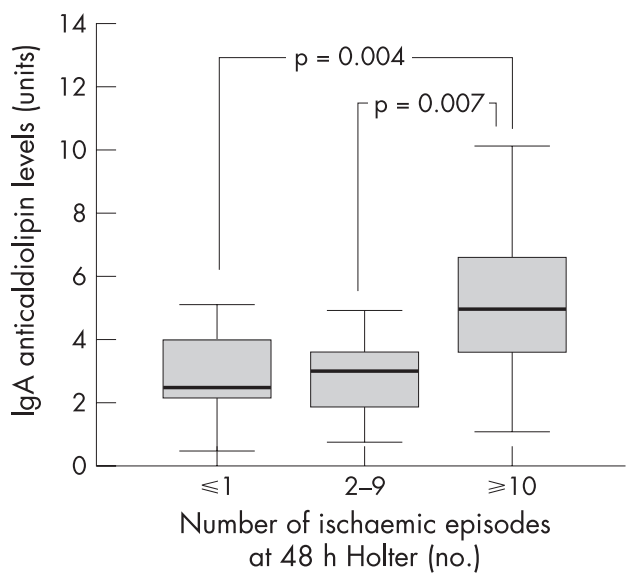

Figure 1 Distribution of $\lg A$ anticardiolipin levels in each tertile of ischaemic episodes $(F=7.1, p=0.002)$. Horizontal lines correspond to median levels of Iga-aCL; $p$ values refer to post-hoc analysis using the Bonferroni correction.

test and correlations by Spearman's rank test. Categorical variables were compared by $\mathrm{X}^{2}$ test.

Patients were divided into three groups, based on the tertiles (T1-T3) of the number or duration of ischaemic episodes during the $\mathrm{HM}(\mathrm{T} 1: \leqslant 1, \mathrm{n}=20 ; \mathrm{T} 2: 2$ to $9, \mathrm{n}=20 ; \mathrm{T} 3$ : $\geqslant 10$, $\mathrm{n}=20 ;$ and $\mathrm{Tl}: \leqslant 1$ sec, $\mathrm{n}=20 ; \mathrm{T} 2,2$ to $31 \mathrm{sec}, \mathrm{n}=20 ; \mathrm{T} 3$, $\geqslant 32$ sec, $\mathrm{n}=20$ ).

All non-normal variables were transformed into ranks and were then analysed as dependent variables by ANOVA (General linear model, SPSS 11.5) in a multivariable model including age, sex, hyperlipidaemia, hypertension, smoking, family history of CAD, blood glucose, BMI, number of diseased vessels, anti-anginal medication, the tertiles of the number or duration of ischaemic episodes and their interaction terms as independent variables. The Bonferroni correction was used for post-hoc comparisons. A value of $\mathrm{p}<0.05$ was considered significant. Receiver operating characteristic curve analysis (ROC) was used to determine the areas under the curves (AUC) of biomarkers for the prediction of the ischaemic burden.

\section{RESULTS}

Patients and controls had similar demographics and distribution of antihypertensive treatment ( $30 \%$ vs $28 \%$ ), while $45 \%$ of both groups were active smokers or had ceased smoking 3 months before inclusion. The patients' subgroups, based on the tertiles of ischaemic episodes, had a similar distribution of anti-anginal treatment. CK and CK-MB levels were normal ( $<195 \mathrm{IU} / \mathrm{l}$ and $<24 \mathrm{IU} / \mathrm{l})$ during $48 \mathrm{~h} \mathrm{HM}$.

Among biomarkers, only IgA-aCL was higher in patients than in controls (3.2 vs 2.4 AEU, $p=0.002$ ). Patients who had more than two ischaemic episodes (2nd-3rd tertile) had higher 11 DHTXB2 than controls $(p=0.01)$. There was no relationship between aCL, 11-DHTXB2, CK or CK-MB.

Only IgA-aCL was related to number $(r=0.51, p=0.008)$ and duration of ischaemic episodes $(r=0.52, p=0.004)$. By ANOVA, patients with $\geqslant 10$ episodes (3rd tertile) or $\geqslant 32 \mathrm{~min}$ ischaemia (3rd tertile) had higher IgA-aCL than those with a lower ischaemic burden (4.95 vs 3 vs $2.5 \mathrm{AEU}, \mathrm{F}=7.1$, $\mathrm{p}=0.002$ and 4.9 vs 3 vs $2.5 \mathrm{AEU}, \mathrm{F}=9.8, \mathrm{p}=0.001$ ) (fig 1 ). Patients with two or more episodes of ischaemia had higher
11-DHTXB2 than those who had less than two episodes (1st tertile) ( 2.9 vs 2.8 vs $1.8 \mathrm{ng} / \mathrm{mg}$ creatinine, $\mathrm{F}=8.3, \mathrm{p}=0.001$ ), even after adjustment for anti-anginal medication.

By ROC, the AUC for IgA-aCL was greater than for 11DHTXB2 in predicting more than 10 episodes (80\%, 95\% CI: 66 to $93, p=0.001$ vs $61 \%, 95 \%$ CI: 52 to $81, p=0.05)$, or $\geqslant 32 \mathrm{~min}$ of ischaemia $(75 \%, 95 \%$ CI: 60 to $88, p=0.004$ vs $58 \%, 95 \%$ CI: 44 to $74, p=0.3)$.

\section{DISCUSSION}

Oxidised low-density lipoprotein may generate aCL. ${ }^{1}$ Ischaemia-induced oxidative stress, C-reactive protein and cytokines may lead to cell apoptosis in atherosclerotic lesions. Apoptotic cells relocate mitochondrial cardiolipins to their cell surface $^{3}$ and may thus trigger aCL production. ${ }^{1}$ IgA-aCL has been linked to chronic vascular inflammation ${ }^{4}$ and is involved in the onset and outcome of acute coronary syndromes. ${ }^{5}$ These mechanisms may explain the elevated IgA-aCL shown in our study.

We have also demonstrated a relationship between IgA-aCL and the extent of ischaemia in the absence of elevated cardiac enzymes.

Myocardial ischaemia may cause endothelial cell apoptosis leading to exposure of mitochondrial cardiolipin, and may enhance the oxidative stress causing an increase in IgA-aCL. Conversely, increases in circulating IgA-aCL may also facilitate ischaemia by promoting endothelial dysfunction, complement activation and inflammation. ${ }^{1}$

The study was not designed to establish whether aCL might be a cause or a consequence of ischaemia or to elucidate the mechanistic insights of the reported correlations. As oxidisedlow density lipoprotein may generate aCL, further studies are required to investigate whether the association between IgAaCL and ischaemia is independent of increases of oxidised-low density lipoprotein.

In conclusion, IgA-aCL is associated with the severity of daily-life ischaemia in chronic CAD. Its utility as a biomarker of ischaemia remains to be determined in large-scale trials.

\section{Authors' affiliations \\ Ignatios Ikonomidis, John Lekakis, Georgia Vamvakou, loanna Revela, Dimitrios T Kremastinos, 2nd Cardiology Department, Attikon Hospital, University of Athens, Greece}

Sozos Loizou, Felicita Andreotti, Petros Nihoyannopoulos, Imperial College School of Medicine, National Heart \& Lung Institute, Cardiology Department, Hammersmith Hospital, London, UK

Competing interests: None declared.

\section{REFERENCES}

1 Mackworth-Young CG. Antiphospholipid syndrome: multiple mechanisms. Clin Exp Immunol 2004;136:393-401.

2 Samarkos M, Davies KA, Gordon C, et al. Clinical significance of IgA anticardiolipin and anti-b2-GP1 antibodies in patients with systemic lupus erythematosus and primary antiphospholipid syndrome. Clin Rheumatol 2006;25: 199-204.

3 Sorice M, Circella A, Cristea Garofalo T, et al. Cardiolipin and its metabolites move from mitochondria to other cellular membranes during death receptor mediated apoptosis. Cell Death Differ 2004;11:1133-45.

4 Tajima C, Suzuki Y, Mizushima Y, et al. Clinical significance of immunoglobulin A antiphospholipid antibodies: possible association with skin manifestations and small vessel vasculitis. J Rheumatol 1998;25:1730-6.

5 Veres K, Lakos G, Kerenyi A, et al. Antiphospholipid antibodies in acute coronary syndrome. Lupus 2004;13:423-75. 